\title{
Luteolin reduces migration of human glioblastoma cell lines via inhibition of the p-IGF-1R/PI3K/AKT/mTOR signaling pathway
}

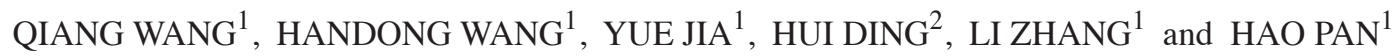 \\ ${ }^{1}$ Department of Neurosurgery, Jinling Hospital, School of Medicine, Nanjing University; ${ }^{2}$ Department of Neurosurgery, \\ Jinling Hospital, School of Medicine, Southern Medical University, Nanjing, Jiangsu 210002, P.R. China
}

Received September 24, 2015; Accepted March 14, 2017

DOI: $10.3892 / \mathrm{ol} .2017 .6643$

\begin{abstract}
Luteolin (3',4',5,7-tetrahydroxyflavone) is a common dietary flavonoid, which has been demonstrated to exert anticancer effects in multiple cancer models. However, the detailed mechanisms underlying the inhibitory effect of luteolin on glioblastoma cell metastasis remain poorly understood. The present study assessed the effects of luteolin in the U251MG and U87MG human glioblastoma cell lines. Luteolin treatment significantly inhibited glioblastoma cell migration, and this effect was associated with downregulated matrix metalloproteinase (MMP)-2, MMP-9 and upregulated tissue inhibitor of metalloproteinase (TIMP)-1 and TIMP-2. In addition, luteolin also inhibited the epithelial-mesenchymal transition-associated phenotype. Furthermore, the phosphorylated insulin-like growth factor-1 receptor/phosphoinositide 3 kinase/protein kinase $\mathrm{B} / \mathrm{mammalian}$ target of rapamycin (p-IGF-1R/PI3K/AKT/mTOR) signaling pathway was demonstrated to participate in these processes. The results of the present study demonstrated that the flavonoid luteolin reduced the migration of glioblastoma cells by altering p-IGF-1R/PI3K/AKT/mTOR activation, and may have potential applications for chemoprevention in a clinical setting.
\end{abstract}

\section{Introduction}

Glioblastoma multiforme (GBM) is the most common malignant primary brain tumors in humans (1). A notable characteristic of GBM is the ability to invade normal, healthy brain tissue, which creates new malignant foci and results in low total resection and a high recurrence rate, which is problematic

Correspondence to: Professor Handong Wang, Department of Neurosurgery, Jinling Hospital, School of Medicine, Nanjing University, 305 East Zhongshan Road, Nanjing, Jiangsu 210002, P.R. China

E-mail: njhdwang@hotmail.com

Key words: luteolin, glioblastoma, migration, epithelial-mesenchymal transition, phosphorylated insulin-like growth factor-1 receptor/phosphoinositide 3-kinase/protein kinase $\mathrm{B} /$ mammalian target of rapamycin in terms of treatment. Due to the high proliferation rate and malignant and invasive characteristics, GBM one of the most aggressive brain tumors, and under the current standard of care the median survival time from diagnosis is 15 months (2). One way to solve this problem would be to effectively inhibit signaling pathways that control cell migration and invasion.

Luteolin (3',4',5,7-tetrahydroxyflavone) is a common dietary flavonoid, which is present at high concentrations in food plants and vegetables (3). Flavonoids are well-known to have effects on xenobiotic and carcinogen metabolism (4). Previous research has demonstrated that plants rich in luteolin have a wide range of antioxidant, anti-inflammatory and anticancer effects (5). Luteolin demonstrates strong anticancer activity against a series of solid tumors, including colonic HT-29, HCT116, hepatic HepG2 and pulmonic A549 (6-9). Although the preclinical anticancer efficacy of luteolin has been demonstrated in various cancer models, its effect on glioblastoma cells has rarely been studied. As a flavonoid, previous studies have revealed that luteolin is able to cross the blood-brain barrier (BBB) $(10,11)$. Furthermore, luteolin is potentially beneficial for the central nervous system (CNS), as it is able to decrease inflammation and axonal damage by preventing monocyte migration across the BBB (12). In the present study, the effects of luteolin on the migration of human glioblastoma cell lines was investigated, and the potential underlying mechanisms were investigated.

\section{Materials and methods}

Cell culture procedures. Human glioblastoma U251MG and U87MG cell lines were obtained from the American Type Culture Collection (Manassas, VA, USA) and cultured in Dulbecco's modified Eagle's medium (DMEM; Gibco; Thermo Fisher Scientific, Inc., Waltham, MA, USA) containing 10\% fetal bovine serum (FBS; Gibco; Thermo Fisher Scientific, Inc.), $100 \mathrm{U} / \mathrm{ml}$ penicillin and $100 \mu \mathrm{g} / \mathrm{ml}$ streptomycin (HyClone; GE Healthcare Life Sciences, Logan, UT, USA). The cells were incubated at $37^{\circ} \mathrm{C}$ in a humidified atmosphere of $95 \%$ air and $5 \% \mathrm{CO}_{2}$.

Reagents and antibodies. Luteolin and phalloidin were purchased from Sigma-Aldrich; Merck KGaA (Darmstadt, Germany). Insulin-like growth factor-1 (IGF-1) was purchased from PeproTech, Inc. (Rocky Hill, NJ, USA) 
Anti-phosphorylated (p-)insulin-like growth factor-1 receptor (IGF-1R) (cat. no. sc-81499; 1:500) was purchased from Santa Cruz Biotechnology, Inc. (Dallas, TX, USA). Anti-matrix metalloproteinase (MMP)-2 (cat. no. ab-7033; 1:1,000), anti-MMP-9 (cat. no. ab-76003; 1:1,000), anti-tissue inhibitor of metalloproteinase (TIMP)-1 (cat. no. ab-109125; 1:1,000) and anti-TIMP-2 (cat. no. ab-157386; 1:1,000) were purchased from Abcam (Cambridge, UK). Anti-E-cadherin, anti-N-cadherin, anti-vimentin, anti- $\beta$-catenin, anti-vimentin (EMT kit; cat. no. cst-9782; 1:1,000), anti-p-protein kinase B (AKT) (cst-4060; 1:1,000), anti-AKT (cat. no. cst-9272; 1:1,000), anti-p-mammalian target of rapamycin (mTOR) (cat. no. cst-2971; 1:1,000), anti-mTOR (cat. no. cst-2983; 1:1,000), anti- $\beta$-actin (cat. no. cst-4970; $1: 1,000$ ), and horseradish peroxidase (HRP)-conjugated goat anti-rabbit immunoglobulin G (IgG; heavy and light chain; cat. no. cst-7074; 1:5,000) secondary antibodies were purchased from Cell Signaling Technology, Inc. (Danvers, MA, USA). The goat anti-mouse IgG-HRP were purchased from BioworldTechnology (cat. no. BS12478; 1:10,000; St. Louis Park, MN, USA).

Cell proliferation assay. Cell proliferation was assessed by Cell Counting Kit-8 (CCK-8) assay (Dojindo Molecular Technologies, Inc., Kumamoto, Japan). Briefly, U251MG and U87MG cells were seeded into 96-well plates at a density of $2 \times 10^{3}$ cells per well, and cultured for at least $24 \mathrm{~h}$ to adhere. Then, the cells were treated with different concentrations of luteolin $(0,5,10,20,40,80$ and $100 \mu \mathrm{M})$. Following treatment for $24 \mathrm{~h}$ at $37^{\circ} \mathrm{C}, 10 \mu \mathrm{l} \mathrm{CCK}-8$ reagent was added to the cells followed by incubation for $2 \mathrm{~h}$ at $37^{\circ} \mathrm{C}$. Then the absorbance was measured at a wavelength of $450 \mathrm{~nm}$ using a Bio-Rad ELISA microplate reader (Bio-Rad Laboratories, Inc., Hercules, CA, USA). The proliferation rate was calculated as follows: [1-optical density (OD) 450 values of treated groups/OD450 values of control group] x $100 \%$.

Scratch-induced migration assay. This was performed as previously described by Etienne-Manneville (13). U251MG and U87MG cells were seeded on collagen-covered 6-well plates at a density of $0.3 \times 10^{6}(\mathrm{U} 251 \mathrm{MG})$ and $0.4 \times 10^{6}(\mathrm{U} 87 \mathrm{MG})$ per plate in DMEM containing 10\% FBS. Following incubation for $24 \mathrm{~h}$, the medium was replaced by DMEM containing $0.5 \% \mathrm{FBS}$, and the cells were treated for $24 \mathrm{~h}$ with $0,5,10$ or $20 \mu \mathrm{M}$ luteolin at $37^{\circ} \mathrm{C}$. In each plate, three areas were scratched, creating three gaps of similar widths with a $200 \mu \mathrm{l}$ standard pipette tip. At the indicated time points $(0,6,12$ and $24 \mathrm{~h})$, phase-contrast images of the plates were obtained using a ZEISS inverted microscope (Zeiss GmbH, Jena, Germany; magnification, $\mathrm{x} 4$ ). The widths of gaps treated with the different concentrations of luteolin and at different time points were measured by Image-Pro Plus software (version 6.0; Media Cybernetics, Inc., Rockville, MD, USA). The widths of the three scratches on each plate were averaged to obtain the mean gap width at a given time. Statistical analysis disclosed the mean gap width (in arbitrary units) of luteolin-treated cells relative to the control (DMSO) at different time points (mean \pm standard error of the mean; $n=3$ ).

Western blot analysis. U251MG and U87MG cells were plated at a density of $0.3 \times 10^{6}(\mathrm{U} 251 \mathrm{MG})$ and $0.4 \times 10^{6}$ (U87MG) cells in 6-well plates or $35 \mathrm{~mm}$ dishes, respectively, and were

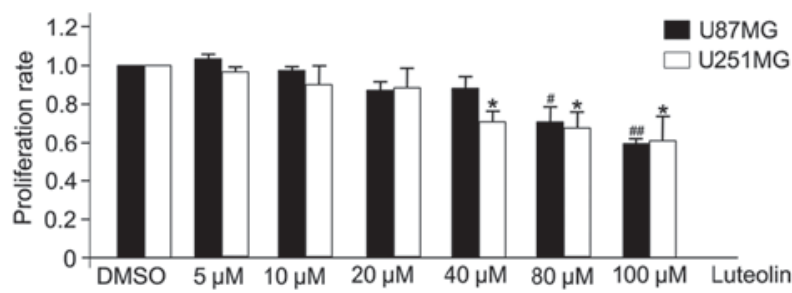

Figure 1. Effects of luteolin on the proliferation of glioblastoma cells U251MG and U87MG cells were treated with luteolin at the indicated concentrations for $24 \mathrm{~h}$, and cell proliferation was analyzed by Cell Counting Kit- 8 assay. All values represent the mean \pm standard error of the mean of three independent experiments. ${ }^{*}, \mathrm{P}<0.05$ and ${ }^{\# \#} \mathrm{P}<0.01$ vs. control cells.

allowed to grow overnight in DMEM containing 10\% FBS. The medium was then replaced by medium without FBS, and the cells were treated for $24 \mathrm{~h}$ with different concentrations of luteolin $(0,5,10$ or $20 \mu \mathrm{M})$ with or without $100 \mathrm{ng} / \mathrm{ml}$ IGF-1. The cells were then lysed with solubilization buffer [50 mmol/l Tris- $\mathrm{HCl}$ (pH 7.6), $20 \mathrm{mmol} / 1 \mathrm{MgCl}_{2}, 200 \mathrm{mmol} / 1$ $\mathrm{NaCl}, 0.5 \% \mathrm{NP} 40,1 \mathrm{mmol} / \mathrm{l}$ DTT and protease inhibitors] on ice for $10 \mathrm{~min}$, and the lysate $(20-100 \mu \mathrm{g})$ was subjected to $10 \%$ sodium dodecyl sulfate-polyacrylamide gel electrophoresis and transferred to polyvinylidene fluoride membranes (Merck KGaA). Following blocking with 5\% nonfat dried milk for $1 \mathrm{~h}$ at room temperature, the membrane was incubated with the appropriate primary antibodies overnight at $4^{\circ} \mathrm{C}$. Then, the immunoreactive bands were visualized using an Enhanced Chemiluminescence kit (EMD Millipore, Billerica, MA, USA) using horseradish peroxidase-conjugated IgG secondary antibodies at room temperature for $2 \mathrm{~h}$.

Cytoskeleton staining. U251MG cells were seeded on glass coverslips in DMEM containing 10\% FBS, placed in 6-well plates at a density of $1 \times 10^{5}$ cells per well, for $24 \mathrm{~h}$. The medium was then replaced by DMEM containing $0.5 \%$ FBS, and $20 \mu \mathrm{M}$ luteolin was added. Following further incubation for $24 \mathrm{~h}$ at $37^{\circ} \mathrm{C}$, the cells were fixed with $4 \%$ paraformaldehyde for $30 \mathrm{~min}$ at room temperature, and were stained using rhodamine-conjugated phalloidin (1:1,000; Sigma-Aldrich; Merck $\mathrm{KGaA}$ ) for $30 \mathrm{~min}$ at room temperature. Slides were washed with PBS, mounted, imaged with 20 fields and counted using a ZEISS fluorescence microscope (Zeiss $\mathrm{GmbH}$ ) (magnification, $x 40$ ). Cells with stress fibers (mean \pm standard error of the mean; $n=3$ ) were expressed as a percentage of 100 cells counted from each slide. Cell area (mean \pm standard error of the mean; $n=3$ ) was measured using ZEN2 software (Zeiss $\mathrm{GmbH})$.

Statistical analysis. Statistical analysis was performed using SPSS 19.0 software (IBM SPSS, Armonk, NY, USA). Data are presented as the mean \pm standard deviation and evaluated using one-way analysis of variance followed with Tukey's post hoc test. $\mathrm{P}<0.05$ was considered to indicate a statistically significant difference.

\section{Results}

Luteolin inhibits the proliferation of glioblastoma cells. Luteolin demonstrated a clear anti-proliferative effect on 
A
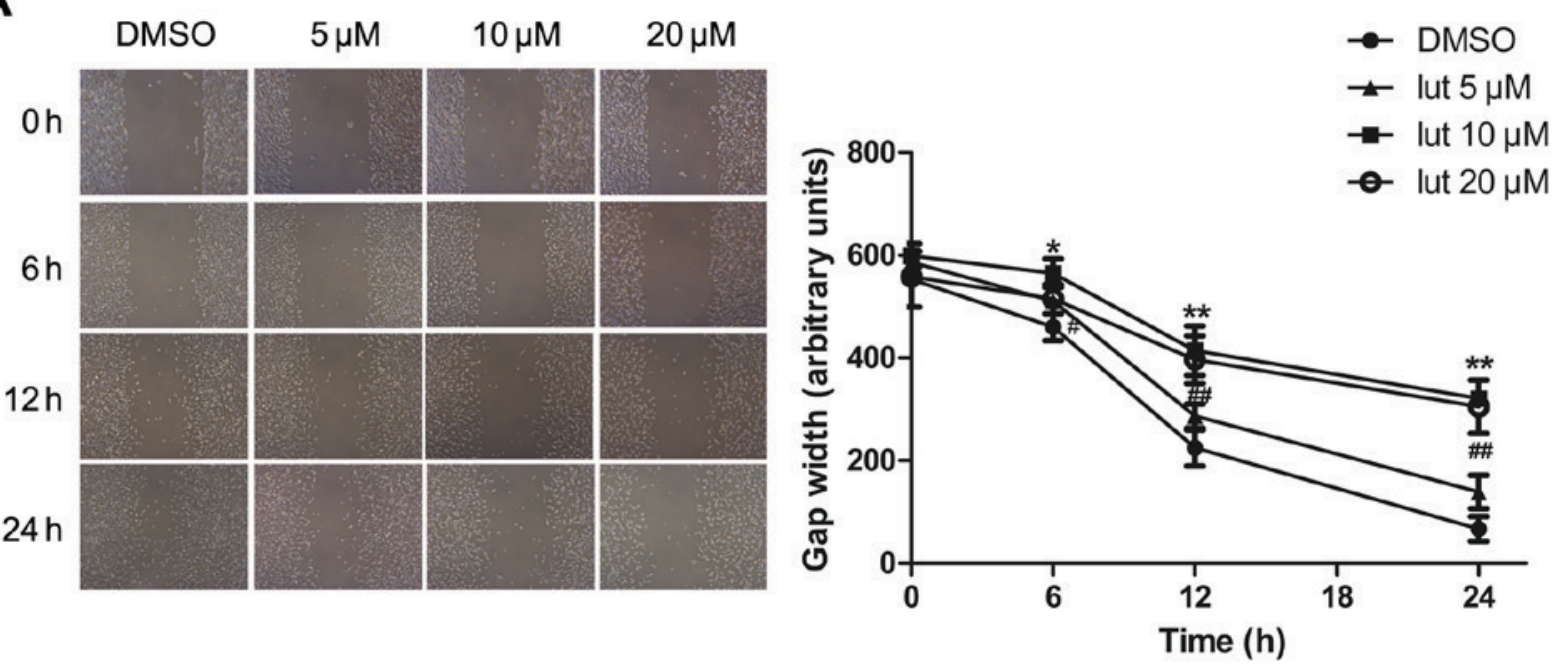

B
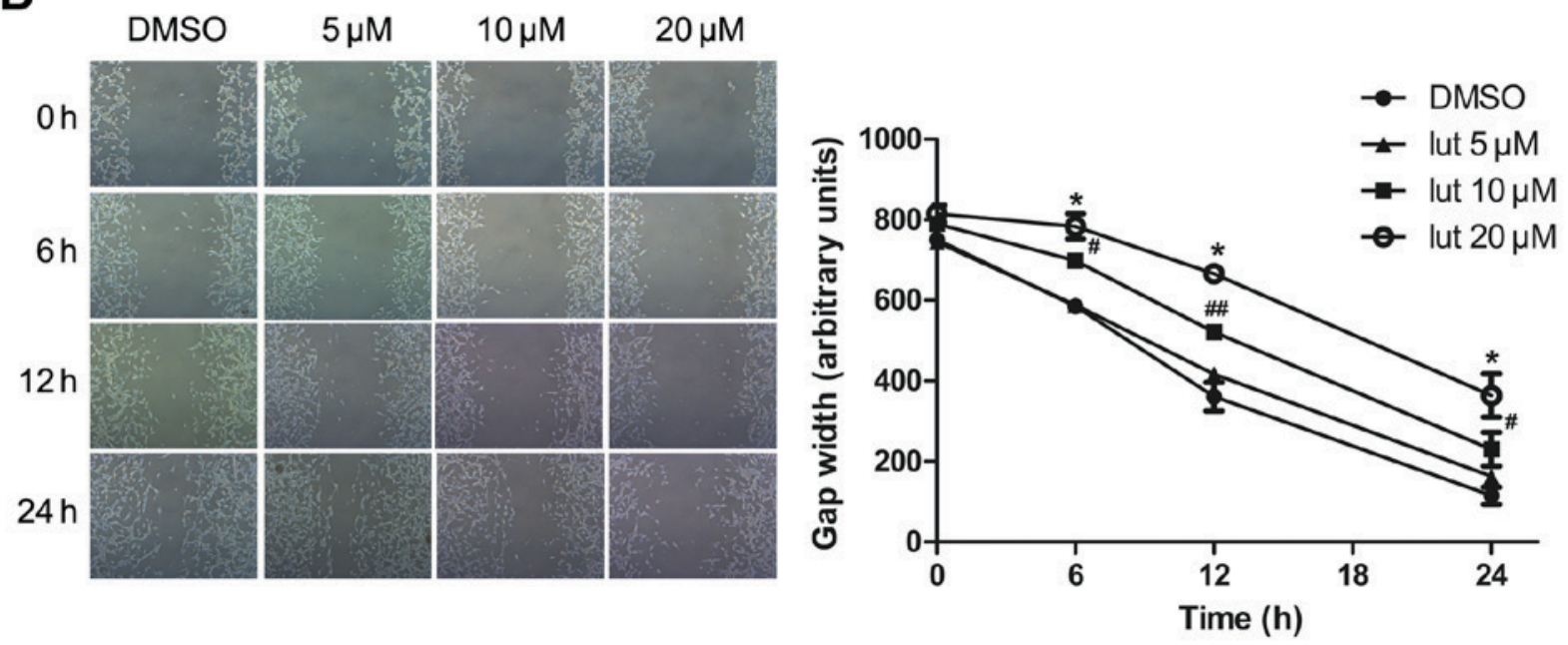

Figure 2. Luteolin disrupted the migration of glioblastoma cells. (A) U251MG and (B) U87MG cells were seeded on collagen-covered six-well plates at a density of $0.3 \times 10^{6}(\mathrm{U} 251 \mathrm{MG})$ and $0.4 \times 10^{6}(\mathrm{U} 87 \mathrm{MG})$ per plate. A scratch wound was inflicted $(0 \mathrm{~h})$, and the resulting gap was imaged and the width measured at different time points $(0,6,12$ and $24 \mathrm{~h})$. All values represent the mean \pm standard error of the mean of three independent experiments. ${ }^{*}, \#<0.05$ and ${ }^{* *}, \# \# P<0.01$ vs. control cells.

U251MG and U87MG cells (Fig. 1). U251MG and U87MG glioblastoma cells were treated with various concentrations of luteolin $(0,5,10,20,40,80$ and $100 \mu \mathrm{M})$ for $24 \mathrm{~h}$, and the effect was examined by CCK- 8 assay. At concentrations $>40 \mu \mathrm{M}$, luteolin significantly inhibited the proliferation of U251MG cells, and significantly inhibited the proliferation of U87MG cells at $80 \mu \mathrm{M}$. However, at concentrations $<20 \mu \mathrm{M}$, there was no apparent anti-proliferative effect. Therefore, to exclude the cytotoxic effects of excess luteolin, the following experiments selected a concentration range of luteolin $<40 \mu \mathrm{M}$ to determine the associated effects on glioblastoma cells.

Luteolin disrupts the migration of glioblastoma cells. Next, the effect of luteolin on the motility of glioblastoma was assessed. This was performed using a scratch-induced migration assay in which the width of the gap formed by a scratch was monitored at different times following the infliction of the wound (13). The U251MG and U87MG cells were pretreated with luteolin $(5,10$ or $20 \mu \mathrm{M})$ or with DMSO (as a control) for
$24 \mathrm{~h}$, and maintained in DMEM containing 0.5\% FBS to block cell proliferation, which would otherwise account for gap closure. The gap width was then monitored at the indicated time points of $0,6,12$ and $24 \mathrm{~h}$. Luteolin treatment significantly decreased the migration ability of glioblastoma cells in a time- and concentration-dependent manner (Fig. 2). There was no difference in width length at the lowest concentration of $5 \mu \mathrm{M}$, whilst at concentrations of 10 and $20 \mu \mathrm{M}$ the gap closure was attenuated. It appeared to move at a slower pace compared with the control cells, closing a smaller portion of the wound gap. Statistical analysis of the results indicated that luteolin caused a significant decrease in U251MG and U87MG cell migration.

Effects of luteolin on the expression of MMP-2, MMP-9, TIMP-1 and TIMP-2 in glioblastoma cells. MMPs are gelatinizes which are capable of degrading the extracelluar matrix (ECM) and facilitating the migration and invasion of cancer cells. In contrast, TIMPs are the endogenous inhibitors of MMPs, and prevent the breakdown of the ECM. Therefore, 
A

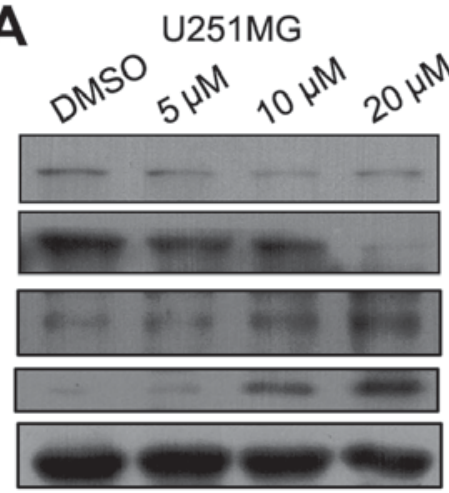

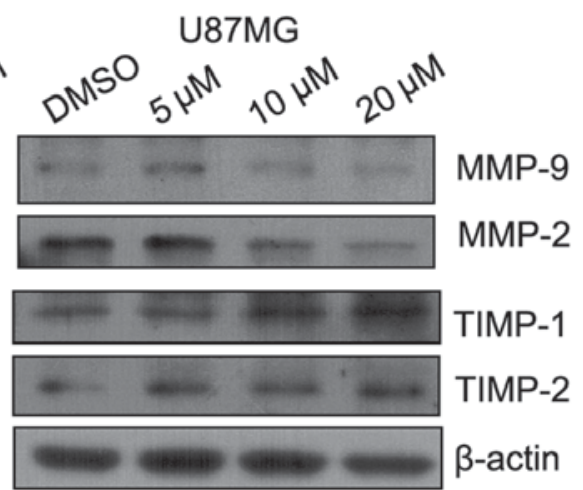

B

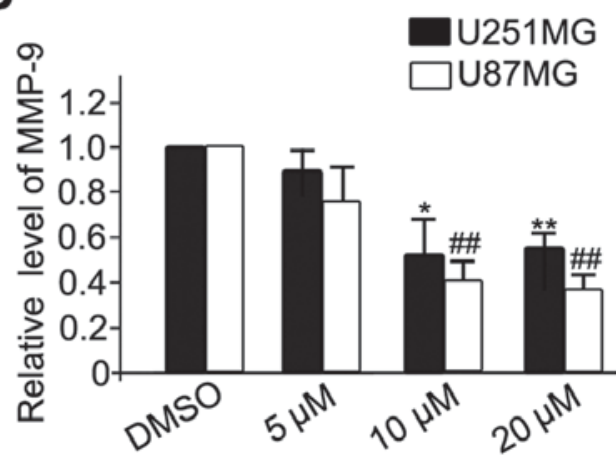

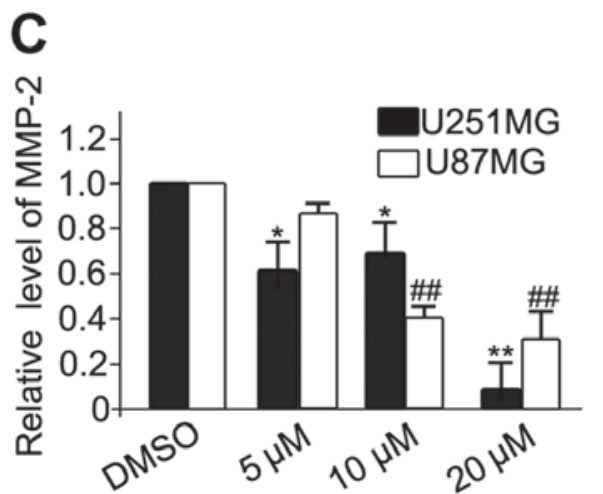

D

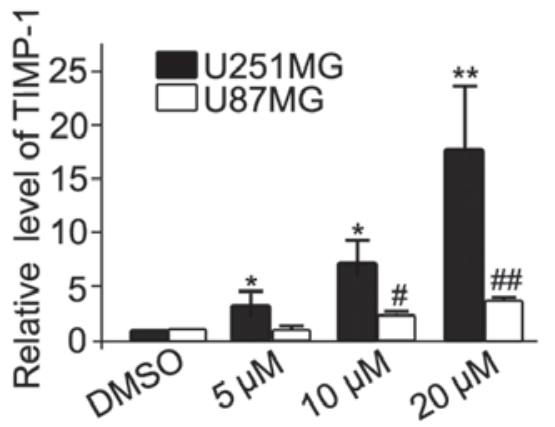

$\mathbf{E}$

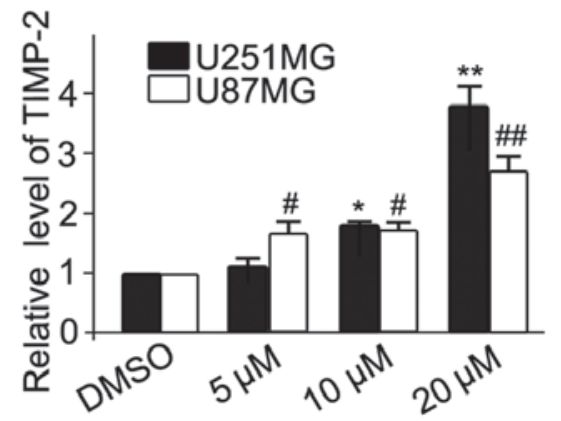

Figure 3. Luteolin decreased the expression of MMP-2 and MMP-9 and increased the expression of TIMP-1 and TIMP-2 in glioblastoma cells. (A) The U251MG and U87MG cells were treated with luteolin $(0,5,10,20 \mu \mathrm{M})$ for $24 \mathrm{~h}$ and then subjected to western blotting. The protein expression levels of (B) MMP-2, (C) MMP-9, (D) TIMP-1 and (E) TIMP-2 were analyzed. $\beta$-actin was used as an internal control. All values represent the mean \pm standard error of the mean of three independent experiments. ${ }^{*}, \mathrm{P}<0.05$ and ${ }^{* *}, \# \# \mathrm{P}<0.01$ vs. control cells. MMP, matrix metalloproteinase; TIMP, tissue inhibitor of metalloproteinase.

the protein expression of MMPs (MMP-2 and MMP-9) and TIMPs (TIMP-1 and TIMP-2) was analyzed (Fig. 3A). The results revealed that luteolin significantly decreased the expression of MMP-2 and MMP-9 (Fig. 3B and C, respectively), and significantly increased the expression of TIMP-1 and TIMP-2 (Fig. 3D and E, respectively). These results demonstrated that luteolin inhibited the migration of glioblastoma cells partially via downregulation of MMPs and upregulation of TIMPs.

Luteolin prevents epithelial-mesenchymal transition (EMT) progression in glioblastoma cells. The process of cancer cell invasion is enabled by EMT, which is the initiator of the metastatic cascade (14). Changes in protein expression levels of E-cadherin, $\mathrm{N}$-cadherin, $\beta$-catenin and Vimentin, all EMT-associated proteins, were assessed. Following luteolin treatment for $24 \mathrm{~h}$, the protein levels of $\mathrm{N}$-cadherin, $\beta$-catenin and Vimentin were visibly decreased; while the protein level of E-cadherin increased (Fig. 4A). A previous study indicated that $\mathrm{N}$-cadherin is upregulated in cancer cells while E-cadherin is downregulated; known as the "cadherin switch' (15). N-cadherin interacts with the fibroblast growth factor receptor, leading to overexpression of MMP-9 and cellular invasion (16). The effects on luteolin on U251MG cell morphology and cytoskeleton organization were then examined using rhodamine-labeled phalloidin, which interacts with polymeric F-actin. The cells were treated with luteolin
$(20 \mu \mathrm{M})$ or with DMSO as a negative control for $24 \mathrm{~h}$ under the same conditions as those used in the scratch-induced migration assay, and then imaged. Control U251MG glioblastoma cells were characterized by small cell bodies and long extensions, while the luteolin-treated cells exhibited flat morphology and were visibly larger (Fig. 4B). In addition, the glioblastoma cells had visibly fewer pseudopodia following luteolin treatment compared with the control (Fig. 4B; as indicated by the green arrow). The majority of the polymeric actin in the control cells appeared to be concentrated in membrane ruffles, while in luteolin-treated cells, polymeric actin was organized into stress fibers (Fig. 4B; as indicated by the white arrow). The cell area of U251MG cells was significantly higher following treatment with $20 \mu \mathrm{M}$ luteolin (Fig. 4C) and the percentage of U251MG cells exhibiting pseudopodia was significantly decreased following treatment with $20 \mu \mathrm{M}$ luteolin (Fig. 4D).

The p-IGF-1R/phosphoinositide 3-kinase (PI3K)/AKT/mTOR signaling pathway is involved in the luteolin-induced reduction in cell migration. The PI3K/AKT/mTOR signaling pathway is involved in regulating the migration of cancer cells. Therefore, the effect of luteolin on this pathway was examined. Luteolin treatment visibly decreased the protein levels of p-AKT and p-mTOR in a concentration-dependent manner (Fig. 5A). IGF-1R is a transmembrane heterotetramer with a cytoplasmic tyrosine kinase domain that activates the PI3K/AKT and 
A

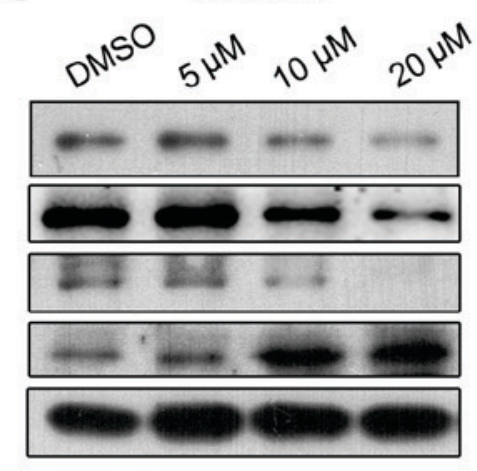

U87MG

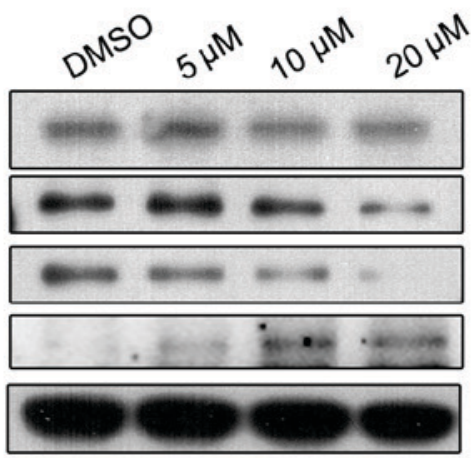

Vimentin

$\beta$-catenin

$\mathrm{N}$-cadherin

E-cadherin

$\beta$-actin
B

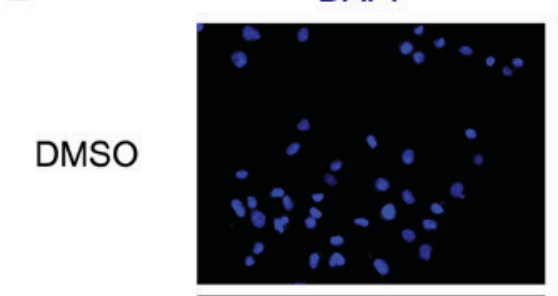

$20 \mu \mathrm{M}$
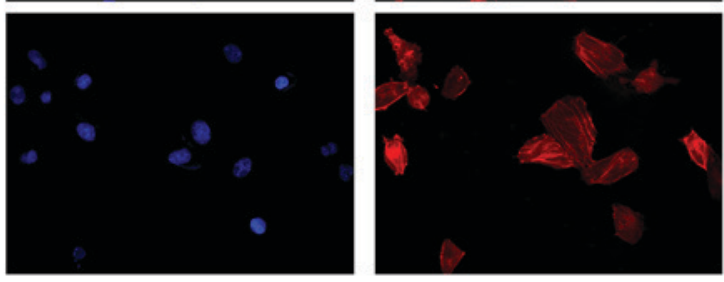

Phalloidin
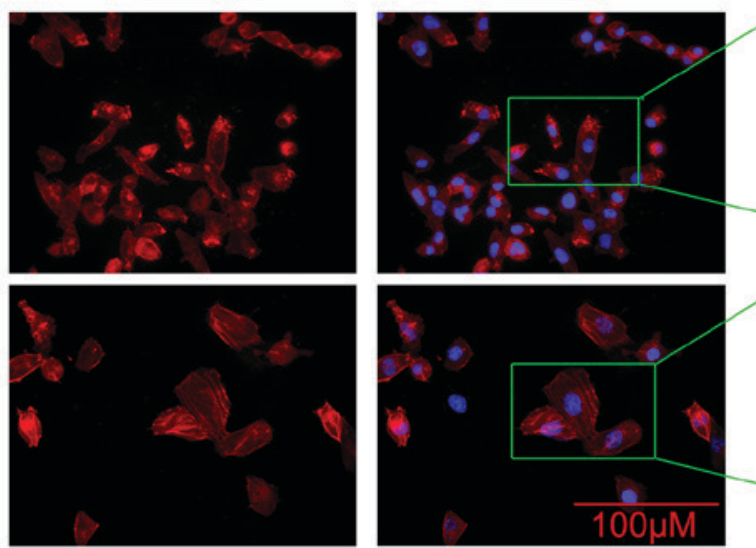

Merge
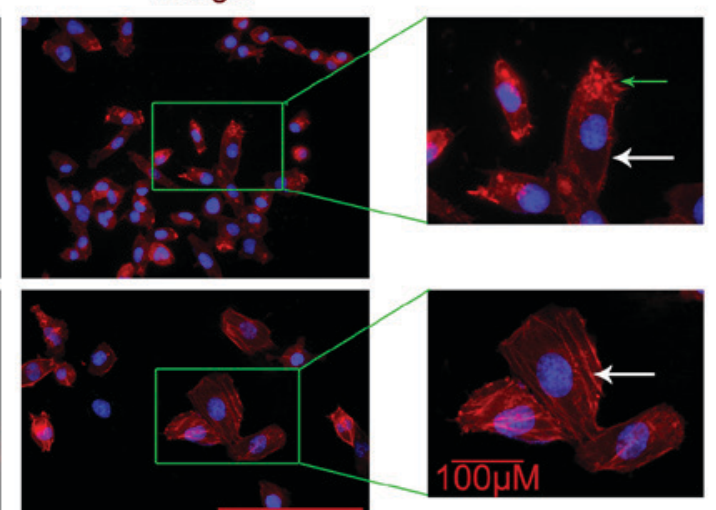

C

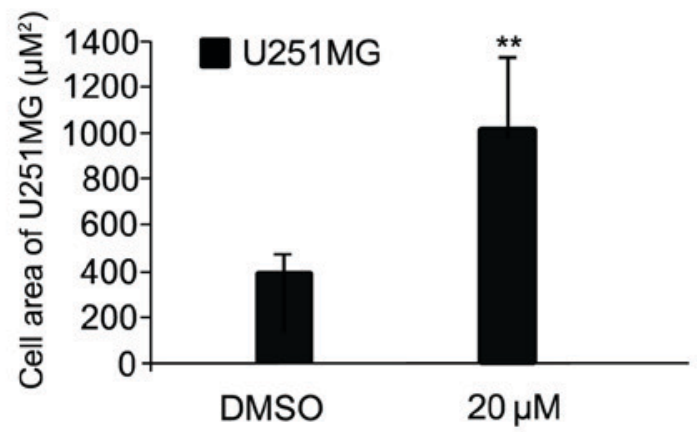

D

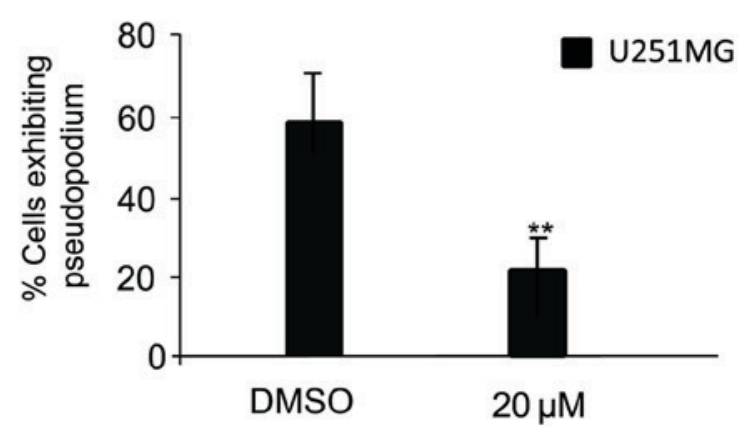

Figure 4. Luteolin prevented EMT progression in glioblastoma cells. (A) Cells were exposed to various concentrations of luteolin for $24 \mathrm{~h}$, and the protein expression levels of Vimentin, $\beta$-catenin, $\mathrm{N}$-cadherin and E-cadherin were determined by western blot. $\beta$-actin was used as an internal control. (B) Representative images of phalloidin-rhodamine (red fluorescence) staining. The green arrow indicates pseudopodia. The white arrows indicate polymeric actin, organized into stress fibers (magnification, $x 40$; scale bar, $100 \mu \mathrm{m}$ ). (C) The cell area and (D) the percentage of cells with pseudopodia were analyzed. All values represent the mean \pm standard error of the mean ${ }^{* *} \mathrm{P}<0.01$ vs. DMSO. EMT, epithelial-mesenchymal transition.

RAS-rapidly accelerated fibrosarcoma-mitogen-activated protein kinase (MAPK) signaling pathways (17). IGF-1R is overexpressed in multiple types of cancer (18). Previous studies have demonstrated that picropodophyllin, an inhibitor of IGF-1R phosphorylation, inhibits the growth of human glioblastoma cell lines and causes tumor regression not only in subcutaneous xenografts, but also in intracerebral xenografts, along with reduced phosphorylation of IGF-1R and AKT (19). Therefore, the protein levels of p-IGF-1R, which was upstream of PI3K, were assessed. These also decreased following treatment with luteolin for $24 \mathrm{~h}$ (Fig. 5A). To further explore the involvement of $\mathrm{p}-\mathrm{IGF}-1 \mathrm{R}$ and this pathway in the anti-migratory effect of luteolin, IGF-1 was used to upregulate p-IGF-1R. The U251MG and U87MG cells were serum-starved overnight and then treated for $24 \mathrm{~h}$ with $20 \mu \mathrm{M}$ luteolin prior to $1 \mathrm{~h}$ stimulation with $100 \mathrm{ng} / \mathrm{ml}$ IGF-1. The results revealed that IGF-1 recovered the level of p-AKT, p-mTOR, MMP-2, MMP-9, N-cadherin and Vimentin, which decreased following treatment of luteolin (Fig. 5B). In addition, the protein levels of TIMP-1, TIMP-1 and E-cadherin also decreased following treatment with IGF-1 (Fig. 5B). These results suggested that luteolin-induced inhibition of migration in glioblastoma cells 
A

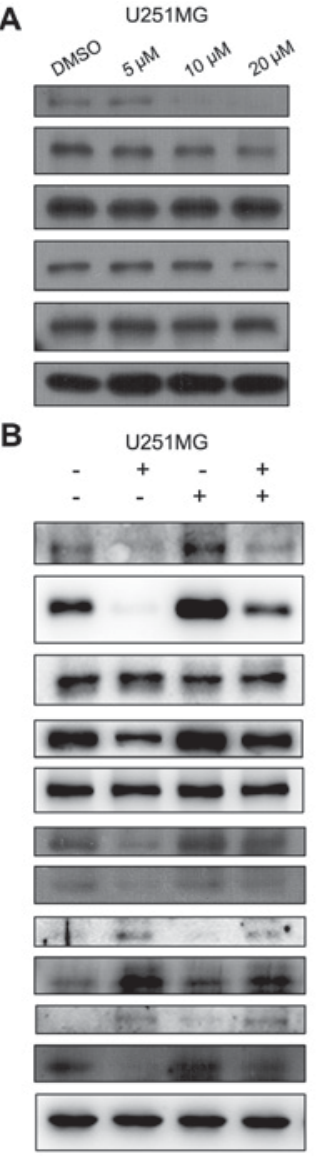

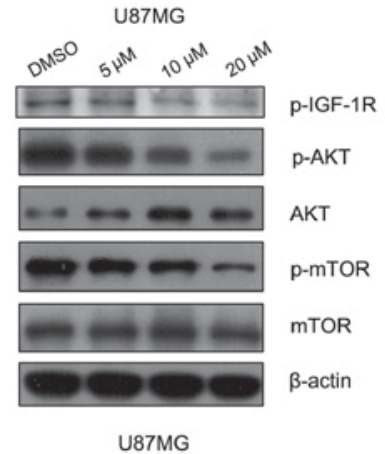

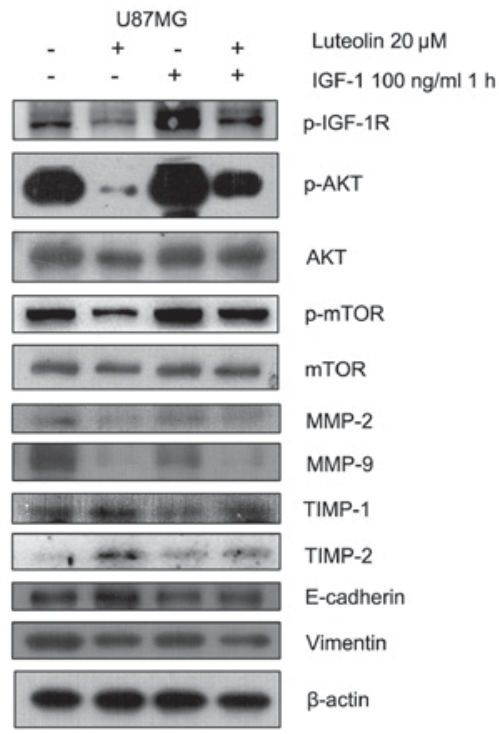

Figure 5. The p-IGF-1R/phosphoinositide 3 kinase/AKT/mTOR signaling pathway was involved in the anti-migration progress of luteolin. (A) Cells were exposed to various concentrations of luteolin for $24 \mathrm{~h}$ and the expression of p-IGF-1R, p-AKT, AKT, p-mTOR and mTOR was detected by western blot. (B) U251MG and U87MG cells were serum-starved overnight and then treated for $24 \mathrm{~h}$ with $20 \mu \mathrm{M}$ luteolin prior to $1 \mathrm{~h}$ stimulation with $100 \mathrm{ng} / \mathrm{ml}$ IGF-1. The expression of p-IGF-1R, p-AKT, AKT, p-mTOR, mTOR, MMP-2, MMP-9, TIMP-1, TIMP-2, E-cadherin, Vimentin and $\beta$-actin was determined. p-, phosphorylated; IGF-1R, insulin-like growth factor-1 receptor; AKT, protein kinase B; mTOR, mammalian target of rapamycin; IGF-1, insulin-like growth factor-1; MMP, matrix metalloproteinase; TIMP, tissue inhibitor of metalloproteinase.

partially occurred through the p-IGF-1R/PI3K/AKT/mTOR signaling pathway.

\section{Discussion}

The present study explored the effect of luteolin on the migration and EMT of human glioblastoma cells. Luteolin was demonstrated to reduce the migration of glioblastoma cells and to weaken the EMT process via suppression of the p-IGF-1R/PI3K/AKT/mTOR signaling pathway. These results provided substantial evidence for the anti-migration and anti-EMT effects of luteolin against human glioblastoma.

The degradation of the vascular basement membrane is required for tumor cells to invade though the basement membrane and migrate to distant secondary sites, and is performed by type IV collagenases known as MMPs $(20,21)$. MMPs are zinc-binding endopeptidases that promote cancer cell migration and invasion via breakdown of the ECM (22). TIMPs are the endogenous inhibitors of the MMPs (23). Therefore, the decreased expression of MMPs and upregulation

of TIMPs may provide a potential therapeutic target to inhibit tumor migration. In the present study, luteolin was demonstrated to decrease MMP-2 and MMP-9 protein levels and increase TIMP-1 and TIMP-2 protein levels. These results suggested that the anti-migratory effect of luteolin was associated with an altered MMP/TIMP balance.

The term EMT describes a process by which stationary epithelial cells lose their characteristic polarity, disassemble their cell-cell junctions and become increasingly motile (24). EMT is considered to be critical event in the process of cancer migration. Thus, the present study analyzed the protein levels of EMT-associated factors, including E-cadherin, $\mathrm{N}$-cadherin, $\beta$-catenin and Vimentin. The protein levels of $\mathrm{N}$-cadherin, $\beta$-catenin and Vimentin decreased following treatment with luteolin, while E-cadherin protein levels increased. The significance of the observed morphological changes lies in the implications for cell motility: Once the actin is organized into stress fibers and focal adhesions are assembled, cells flatten and become attached to the ECM (25). Cells in motion need to assemble and disassemble actin structures to progress, alternating between attachment to and detachment from the ECM. Luteolin treatment induced alterations in cell morphology, which were attributed to reorganization of the actin cytoskeleton. These results are important when contemplating novel treatments for human glioblastoma.

IGF-1 R is a transmembrane heterotetramer with a cytoplasmic tyrosine kinase domain that activates the PI3K/AKT and RAS/MAPK signaling pathways. IGF-1R overexpression is a characteristic common to multiple types of human cancer (26). IGF-1R has previously been suggested to be involved in cerebellum tumors and in neuroblastomas $(27,28)$. Furthermore, the cyclolignan picropodophyllin, a specific inhibitor of IGF-1R, inhibits the growth of human GBM cell lines along with reduced phosphorylation of IGF-1R and AKT (29). In addition, the PI3K/AKT/mTOR signaling pathway controls the migration of cancer cells, and anticancer drugs inhibiting the PI3K/AKT/mTOR axis reduce cell migration (30). The present study demonstrated that luteolin decreased the phosphorylation of IGF-1R, AKT and mTOR in a concentration-dependent manner. The specific activator of IGF-1R, IGF-1, was able to recover the decreased protein level of MMP-2, MMP-9 and Vimentin and decrease the level of TIMP-1 and TIMP-2, suggesting that the $\mathrm{p}-\mathrm{IGF}-1 \mathrm{R} / \mathrm{PI} 3 \mathrm{~K} / \mathrm{AKT} / \mathrm{mTOR}$ signaling pathway was associated with luteolin-mediated inhibition of migration.

Taken together, the results of the present provided evidence that luteolin exerted anti-migratory and anti-EMT effects in human glioblastoma cells via inhibition of the p-IGF-1R/PI3K/AKT/mTOR signaling pathway. These results suggest that luteolin may be attractive therapeutic agent for the development of future treatment protocols.

\section{Acknowledgements}

The authors would like to thank Dr Han Yanling (Department of Neurosurgery, Jinling Hospital, School of Medicine, Nanjing University, Nanjing, China) for the technical assistance. The present study was supported by grants from the National Natural Science Foundation of China (grant 
no. 81371357) and the China Postdoctoral Science Foundation (grant no. 2014M562665, 2015T81136).

\section{References}

1. Ostrom QT, Gittleman H, Farah P, Ondracek A, Chen Y, Wolinsky Y, Stroup NE, Kruchko C and Barnholtz-Sloan JS: CBTRUS statistical report: Primary brain and central nervous system tumors diagnosed in the United States in 2006-2010. Neuro Oncol 15 (Suppl 2): ii1-ii56, 2013.

2. Wen PY and Kesari S: Malignant gliomas in adults. N Engl J Med 359: 492-507, 2008.

3. Lin Y, Shi R, Wang X and Shen HM: Luteolin, a flavonoid with potentials for cancer prevention and therapy. Curr Cancer Drug Targets 8: 634-646, 2008.

4. Moon YJ, Wang X and Morris ME: Dietary flavonoids: Effects on xenobiotic and carcinogen metabolism. Toxicol In Vitro 20 187-210, 2006

5. Chian S, Thapa R, Chi Z, Wang XJ and Tang X: Luteolin inhibits the Nrf2 signaling pathway and tumor growth in vivo. Biochem Biophys Res Commun 447: 602-608, 2014.

6. Chang J, Hsu Y, Kuo P, Kuo Y, Chiang L and Lin C: Increase of $\mathrm{Bax} / \mathrm{Bcl}-\mathrm{XL}$ ratio and arrest of cell cycle by luteolin in immortalized human hepatoma cell line. Life Sci 76: 1883-1893, 2005.

7. Lee HJ, Wang CJ, Kuo HC, Chou FP, Jean LF and Tseng TH: Induction apoptosis of luteolin in human hepatoma HepG2 cells involving mitochondria translocation of Bax/Bak and activation of JNK. Toxicol Appl Pharmacol 203: 124-131, 2005.

8. Lim DY, Jeong Y, Tyner AL and Park JH: Induction of cell cycle arrest and apoptosis in HT-29 human colon cancer cells by the dietary compound luteolin. Am J Physiol Gastrointest Liver Physiol 292: G66-G75, 2007.

9. Tang X, Wang H, Fan L, Wu X, Xin A, Ren H and Wang XJ Luteolin inhibits Nrf2 leading to negative regulation of the Nrf2/ARE pathway and sensitization of human lung carcinoma A549 cells to therapeutic drugs. Free Radic Biol Med 50 $1599-1609,2011$.

10. Youdim KA, Qaiser MZ, Begley DJ, Rice-Evans CA and Abbott NJ: Flavonoid permeability across an in situ model of the blood-brain barrier. Free Radic Biol Med 36: 592-604, 2004.

11. Jang S, Kelley KW and Johnson RW: Luteolin reduces IL-6 production in microglia by inhibiting JNK phosphorylation and activation of AP-1. Proc Natl Acad Sci USA 105: 7534-7539, 2008.

12. Hendriks JJ, Alblas J, van der Pol SM, van Tol EA, Dijkstra CD and de Vries HE: Flavonoids influence monocytic GTPase activity and are protective in experimental allergic encephalitis J Exp Med 200: 1667-1672, 2004.

13. Etienne-Manneville S: In vitro assay of primary astrocyte migration as a tool to study Rho GTPase function in cell polarization. Methods Enzymol 406: 565-578, 2006.

14. Subramani R, Lopez-Valdez R, Arumugam A, Nandy S, Boopalan $\mathrm{T}$ and Lakshmanaswamy R: Targeting insulin-like growth factor 1 receptor inhibits pancreatic cancer growth and metastasis. PLoS One 9: e97016, 2014.
15. Aigner K, Dampier B, Descovich L, Mikula M, Sultan A, Schreiber M, Mikulits W, Brabletz T, Strand D, Obrist P, et al: The transcription factor ZEB1 (deltaEF1) promotes tumour cell dedifferentiation by repressing master regulators of epithelial polarity. Oncogene 26: 6979-6988, 2007.

16. Hazan RB, Qiao R, Keren R, Badano I and Suyama K: Cadherin switch in tumor progression. Ann N Y Acad Sci 1014: 155-163, 2004.

17. Baserga R, Peruzzi F and Reiss K: The IGF-1 receptor in cancer biology. Int J Cancer 107: 873-877, 2003.

18. King H, Aleksic T, Haluska P and Macaulay VM: Can we unlock the potential of IGF-1R inhibition in cancer therapy? Cancer Treat Rev 40: 1096-1105, 2014.

19. Yin S, Girnita A, Strömberg T, Khan Z, Andersson S, Zheng H, Ericsson C, Axelson M, Nistér M, Larsson O, et al: Targeting the insulin-like growth factor-1 receptor by picropodophyllin as a treatment option for glioblastoma. Neuro Oncol 12: 19-27, 2010.

20. Kim YS, Lee HA, Lim JY, Kim Y, Jung CH, Yoo SH and Kim Y: $\beta$-Carotene inhibits neuroblastoma cell invasion and metastasis in vitro and in vivo by decreasing level of hypoxia-inducible factor-1 $\alpha$. J Nutr Biochem 25: 655-664, 2014.

21. Hiraoka N, Allen E, Apel IJ, Gyetko MR and Weiss SJ: Matrix metalloproteinases regulate neovascularization by acting as pericellular fibrinolysins. Cell 95: 365-377, 1998

22. Lu KW, Chen JC, Lai TY, Yang JS, Weng SW, Ma YS, Lu PJ, Weng JR, Chueh FS, Wood WG and Chung JG: Gypenosides inhibits migration and invasion of human oral cancer SAS cells through the inhibition of matrix metalloproteinase-2 -9 and urokinase-plasminogen by ERK1/2 and NF-kappa B signaling pathways. Hum Exp Toxicol 30: 406-415, 2011.

23. Brew K, Dinakarpandian D and Nagase H: Tissue inhibitors of metalloproteinases, evolution, structure and function. Biochim Biophys Acta 1477: 267-283, 2000.

24. Thiery JP and Sleeman JP: Complex networks orchestrate epithelial-mesenchymal transitions. Nat Rev Mol Cell Biol 7: 131-142, 2006.

25. Raftopoulou M and Hall A: Cell migration: Rho GTPases lead the way. Dev Biol 265: 23-32, 2004.

26. Clemmons DR: Modifying IGF1 activity: An approach to treat endocrine disorders, atherosclerosis and cancer. Nat Rev Drug Discov 6: 821-833, 2007.

27. Wojtalla A, Salm F, Christiansen DG, Cremona T, Cwiek P, Shalaby T, Gross N, Grotzer MA and Arcaro A: Novel agents targeting the IGF-1R/PI3K pathway impair cell proliferation and survival in subsets of medulloblastoma and neuroblastoma. PLoS One 7: e47109, 2012.

28. Wang JY, Del Valle L, Gordon J, Rubini M, Romano G, Croul S, Peruzzi F, Khalili K and Reiss K: Activation of the IGF-IR system contributes to malignant growth of human and mouse medulloblastomas. Oncogene 20: 3857-3868, 2001.

29. Yin S, Girnita A, Stromberg T, Khan Z, Andersson S, Zheng H, Ericsson C, Axelson M, Nistér M, Larsson O, et al: Targeting the insulin-like growth factor-1 receptor by picropodophyllin as a treatment option for glioblastoma. Neuro Oncol 12: 19-27, 2010.

30. Cheng CK, Fan QW and Weiss WA: PI3K signaling in glioma-animal models and therapeutic challenges. Brain Pathol 19: 112-120, 2009. 